\title{
RESÍDUO PIRITOSO PROVOCA TOXICIDADE AGUDA E CRÔNICA EM COLLEMBOLA E OLIGOCHAETA
}

\author{
Pyritic waste causes chronic and acute toxicity in Collembola and Oligochaeta
}

Luís Carlos Iuñes de Oliveira Filho ${ }^{1,2 *}$, Dilmar Baretta ${ }^{3}$, Talyta Zortéa ${ }^{4}$, Jaqueline Pereira Machado de Oliveira ${ }^{2}$, Julio Cesar Pires Santos ${ }^{1}$

1. Universidade do Estado de Santa Catarina, Centro de Ciências Agroveterinárias (UDESC-CAV), Departamento de Solos e Recursos Naturais, Programa de Pós-graduação em Ciência do Solo, Lages, Santa Catarina, Brasil. E-mail: iunes1981@gmail.com (*autor para correspondência), julio.santos@udesc.br

2. Universidade do Oeste de Santa Catarina (UNOESC), Departamento de Agronomia. E-mail: jaqueline.oliveira@unoesc.edu.br 3. Universidade do Estado de Santa Catarina, Centro de Educação Superior do Oeste (UDESC Oeste), Departamento de Zootecnia, Chapecó, Santa Catarina, Brasil. Bolsista em Produtividade Científica CNPq. E-mail: dilmar.baretta@udesc.br 4. Universidade do Estado de Santa Catarina, Centro de Educação Superior do Oeste (UDESC Oeste), Departamento de Zootecnia, Programa de Pós-graduação em Zootecnia, Chapecó, Santa Catarina, Brasil. E-mail: talizortea@hotmail.com

Artigo enviado em 16/01/2017, aceito em 08/02/2017 e publicado em 11/04/2017.

RESUMO: A presença de resíduo piritoso no solo afeta negativamente a meso e macrofauna edáfica. Ainda assim, há uma falta de conhecimento sobre como o resíduo pode afetar a biota do solo. O objetivo desta pesquisa foi avaliar as respostas agudas e crônicas em Folsomia candida e Eisenia andrei, após exposição ao resíduo piritoso (carvão mais a pirita) nas doses de 0; 2,5; 5; 10 e 20\% em um Argissolo Vermelho-Amarelo e um Nitossolo Háplico. Para tal, foram realizados testes de fuga, sobrevivência e reprodução com os organismos. Os resultados encontrados mostram que o valor do $\mathrm{pH}$ diminuiu com a aplicação do resíduo piritoso. Todas as concentrações avaliadas levaram a fuga de $F$. candida e E. andrei de ambos os solos contaminados. Os valores de $\mathrm{CF}_{50}$ (concentração estimada que origina $50 \%$ de fuga) ficaram abaixo de 5,3\% para F. candida e de 8,5\% para E. andrei. A sobrevivência de F. candida foi afetada no Argissolo e no Nitossolo, enquanto E. andrei foi afetada apenas no Argissolo. Ambos organismos obtiveram uma baixa reprodução e em alguns casos não ocorrendo reprodução. Nos testes de sobrevivência e reprodução de $F$. candida o valor de $\mathrm{CL}_{50}$ (concentração estimada que causa 50\% de letalidade dos organismos) ficou abaixo $6,9 \% \mathrm{e}$ $\mathrm{CE}_{50}$ (efeitos negativos na reprodução) foi inferior a 2,5\%. Para E. andrei, os valores de $\mathrm{CL}_{50}$ 18,7\% no Argissolo e $>20 \%$ no Nitossolo e o valor de $\mathrm{CE}_{50}$ abaixo de 5,5\%. Concentrações testadas neste estudo causaram efeitos negativos e representam informações ecotoxicológicas importantes para avaliações de risco ecológico de áreas ligadas a mineração de carvão.

Palavras-Chave - Pirita. Ecotoxicologia terrestre. Folsomia candida. Eisenia andrei.

Abstract - Studies indicate that presence of pyritic waste on soil affects the edaphic meso and macrofaunal. Still, there is a lack of knowledge about how this waste can affect the soil biota. The objective of this research was to evaluate the acute and chronic responses in Folsomia candida and Eisenia andrei after exposure pyritic waste (coal plus pyrite) at doses of $0 ; 2.5 ; 5 ; 10$ and 20\% in Ultisol and Hapludox. To this, avoidance tests, survival, and reproduction with the organisms were performed. The results show that the $\mathrm{pH}$ value decreased with the application of pyritic waste. All concentrations evaluated led to avoidance F. candida and E. andrei from both contaminated soil. The $\mathrm{AC}_{50}$ values (estimated concentration which causes 50\% avoidance) were below $5.3 \%$ for F. candida and $8.5 \%$ for E. andrei. F. candida survival was affected in Ultisol and Hapludox, while E. andrei was affected only in Ultisol but not in Hapludox. Both organisms obtained a low reproduction and in some cases not occurring reproduction. In tests of survival and reproduction of F. candida the value of $\mathrm{LC}_{50}$ (estimated concentration that causes $50 \%$ of lethality of organisms) was lower $6.9 \%$ and $\mathrm{EC}_{50}$ (negative for reproduction) was less than $2.5 \%$. For E. andrei, the $\mathrm{LC}_{50}$ values of $18.7 \%$ in the Ultisol and $>20 \%$ in Hapludox and the $\mathrm{EC}_{50}$ value below $5.5 \%$. Concentrations tested in this study cause negative effects and represent important ecotoxicological information for ecological risk assessments of areas related to coal mining.

Keywords - Pyrite. Soil ecotoxicology. Folsomia candida. Eisenia andrei. 


\section{INTRODUÇÃO}

A contaminação do ambiente gera riscos significantes para a saúde humana e aos ecossistemas terrestres e aquáticos (Kostarelos et al., 2015). Uma fonte potencial de poluição é o efluente de drenagem ácida de mina (DAM), que é ocasionado pela oxidação de sulfetos (por exemplo, a pirita - $\mathrm{FeS}_{2}$ ), e por consequência gera águas ácidas com elevadas concentrações de $\mathrm{SO}_{4}^{-2}$, $\mathrm{Fe}, \mathrm{Al}, \mathrm{Mn}$ e numerosos metais tóxicos específicos da mineralogia do minério extraído (Simmons, 2010). Esse resíduo rico em sulfetos é frequentemente encontrado em locais de mineração de carvão e representa sérios problemas ambientais, que ocorrem durante a exploração de carvão e em corpos de resíduos depositados ao longo das áreas de mineração (Bhuiyan et al., 2010).

A disposição inapropriada de resíduos provenientes da atividade de mineração causa a contaminação do solo e consideráveis danos nos ecossistemas, podendo resultar na perda de algumas ou várias funções essenciais do solo. Visto que, a disposição deste material gera problemas ambientais, a DAM tornou-se visível ao longo dos anos e uma série de pesquisas têm avaliado seus efeitos em solos já contaminados por sulfetos (Alvarenga et al., 2012; Lortzie et al., 2015).

Nos últimos anos, a pesquisa em relação aos riscos de contaminação por resíduos de mineração tem ganhado expressividade na comunidade científica brasileira. Vários trabalhos avaliando diferentes atributos de solos na região sul de Santa Catarina vem sendo realizados (Lunardi Neto et al., 2008; Oliveira Filho et al., 2015a; Rodriguez-Iruretagoiena et al., 2015), além de avaliações na qualidade da água (Pompêo et al., 2004) e do próprio carvão (Silva et al., 2011; Oliveira et al., 2012; Francisconi et al., 2013). Entretanto, apesar de vários anos de pesquisa, ainda há poucos estudos que avaliam o comportamento da meso e macrofauna edáfica em relação ao resíduo da mineração, tratado ou não, aplicado no solo (Geremias et al., 2012; Oliveira Filho et al., 2015b).

Assim, torna-se essencial uma avaliação ambiental mais profunda dos efeitos da contaminação de resíduos da mineração de carvão sobre a biota do solo em áreas agrícolas no entorno das áreas mineradas ou em áreas recuperadas e determinar quais os possíveis riscos para os organismos expostos a este material. Uma importante forma de avaliação são os ensaios ecotoxicológicos, que têm sido amplamente utilizados para estimar os efeitos prejudiciais em organismos expostos aos contaminantes ambientais.

A Ecotoxicologia utiliza de importantes ferramentas para avaliação dos efeitos causados por substâncias químicas em diferentes organismos e populações, fornecendo subsídios para avaliar a qualidade de corpos receptores e proteger adequadamente a qualidade ambiental. Caracteriza-se pela associação das concentrações de contaminantes no ambiente e seu possível risco ecotoxicológico, servindo como complementação dos mecanismos tradicionais do controle da poluição, como é o caso das caracterizações químicas (Chapman, 2002). Diversos organismos do solo têm sido utilizados em estudos ecotoxicológicos com a finalidade de avaliar os impactos ambientais, incluindo meso (como colêmbolos) e macrofauna (como minhocas).

Colêmbolos (Collembola) vivem em números elevados na camada superficial do solo alimentando-se de hifas fúngicas e matéria orgânica (Hopkin, 1997), onde desempenham um papel importante na decomposição do material orgânico e consequentemente na ciclagem de nutrientes. As minhocas (Oligochaeta) desempenham um papel vital na manutenção da fertilidade do solo mediante o cumprimento de numerosas funções vitais para o ecossistema, tais como mistura de camadas de solo, degradação da matéria orgânica e aeração do solo (Arnold e Hodson, 2007). Em ensaios ecotoxicológicos, esses grupos são representados principalmente pelas espécies Folsomia candida e Eisenia andrei que são organismos testes adequados e largamente utilizados.

Estes organismos são importantes bioindicadores de qualidade do solo e são capazes de indicar diferentes níveis de contaminação do ambiente em que vivem. Além disso, o solo é um dos principais compartimentos para avaliação do risco ambiental e humano, uma vez que, possui capacidade de fornecer serviços ecossistêmicos em termos de produção de alimentos, como "reservatório" de biodiversidade e como um regulador de gases, água e nutrientes. Para reduzir as pressões ambientais, é essencial conhecer quais as implicações de um contaminante, possibilitando a melhoria na gestão de resíduos e para proteção dos recursos terrestres e hídricos.

Neste contexto, o objetivo deste estudo foi obter informações sobre o efeito da adição de resíduo piritoso gerado da mineração de carvão sobre um representante da meso (colêmbolos) e outro da macrofauna edáfica (minhocas) com a finalidade de 
determinar o risco ecotoxicológico, como forma de prever o impacto biológico da deposição deste material no solo.

\section{MATERIAL E MÉTODOS}

\section{Descrição do resíduo, solo e tratamentos}

O resíduo piritoso no presente estudo foi considerado como pirita $\left(\mathrm{FeS}_{2}\right)$ mais carvão mineral, embora outros minerais possam contribuir para a DAM, entre os Fe-minerais presentes em toda região sul de Santa Catarina a maior abundância deve-se a pirita, como verificado por Silva et al. (2011). A coleta ocorreu em um "lavatório" de carvão no município de Lauro Müller $\left(28^{\circ} 22^{\prime} 20.13^{\prime \prime S}, 4^{\circ} 26^{\prime} 04.92^{\prime \prime O}\right)$ no Sul de Santa Catarina em janeiro de 2012, o qual esteve estocado em pilhas de 2-3 $\mathrm{m}$ por três anos. Foram feitas 10 amostragens, coletando em média 2 $\mathrm{kg}$ do resíduo piritoso de 5 pilhas. Para melhor homogeneização do material, as amostras foram trituradas, peneiradas (malha de $1,25 \mathrm{~mm}$ ) e armazenadas em recipientes fechados hermeticamente para reduzir o processo de oxidação do material.

Foram selecionados dois solos subtropicais de texturas diferentes como solos referência sem histórico de uso agrícola e de contaminação de pirita para evitar efeitos de contaminantes que não o do resíduo piritoso aplicado. Foi coletado um Argissolo Vermelho Amarelo em Lauro Müller na borda de um remanescente de mata $\left(28^{\circ} 23^{\prime} 28.40 " \mathrm{~S}\right.$, $\left.49^{\circ} 28^{\prime} 12.16 " \mathrm{O}\right)$, distante aproximadamente $4 \mathrm{~km}$ do local onde foi coletado o resíduo; e um Nitossolo Háplico em Capão Alto na Serra Catarinense, em área coberta com gramíneas nativas a beira da rodovia SC-

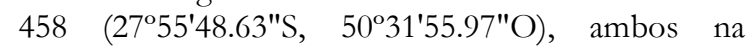
camada de $0-10 \mathrm{~cm}$. Os solos foram previamente peneirados (malha de $4 \mathrm{~mm}$ ) e desfaunados (com dois ciclos de congelamento a $-20{ }^{\circ} \mathrm{C}$ e descongelamento em temperatura ambiente, $24 \mathrm{~h} / 24 \mathrm{~h})$. A caracterização dos solos e do resíduo é apresentada no Tabela 1 .

Tabela 1. Caracterização física e química do Argissolo Vermelho Amarelo e Nitossolo Háplico e do resíduo piritoso utilizado nos testes (Oliveira Filho et al., 2015b).

\begin{tabular}{|c|c|c|c|c|c|}
\hline Atributo & Argissolo & Nitossolo & Resíduo piritoso & $\operatorname{LOD}^{(8)}$ & $\operatorname{LOQ}^{(9)}$ \\
\hline pH água ${ }^{(1)}$ & 3,55 & 4,48 & $\mathrm{ND}$ & & \\
\hline $\operatorname{MOS}(\%)^{(2)}$ & 4,70 & 1,50 & $\mathrm{ND}$ & & \\
\hline CTC $\mathrm{pH} 7,0\left(\mathrm{cmol}_{\mathrm{c}} \mathrm{dm}^{-3}\right)^{(3)}$ & 45,39 & 35,23 & ND & & \\
\hline $\mathrm{Al}^{3+}\left(\mathrm{cmol}_{\mathrm{c}} \mathrm{dm}^{-3}\right)^{(4)}$ & 5,76 & 4,11 & $\mathrm{ND}$ & & \\
\hline $\mathrm{H}+\mathrm{Al}\left(\mathrm{cmol}_{\mathrm{c}} \mathrm{dm}^{-3}\right)^{(5)}$ & 43,30 & 30,70 & $\mathrm{ND}$ & & \\
\hline Argila $\left(\mathrm{g} \mathrm{kg}^{-1}\right)^{(6)}$ & $200(20 \%)$ & $160(16 \%)$ & ND & & \\
\hline Silte $\left(\mathrm{g} \mathrm{kg}^{-1}\right)^{(6)}$ & $500(50 \%)$ & $370(37 \%)$ & ND & & \\
\hline Areia $\left(\mathrm{g} \mathrm{kg}^{-1}\right)^{(6)}$ & $300(30 \%)$ & $470(47 \%)$ & $\mathrm{ND}$ & & \\
\hline \multicolumn{6}{|l|}{ Total $(7)$} \\
\hline $\mathrm{Cu}\left(\mathrm{mg} \mathrm{kg}^{-1}\right)$ & $108 \pm 1,58$ & $26 \pm 0,56$ & $70 \pm 29,50$ & 0,01 & 0,05 \\
\hline $\mathrm{Zn}\left(\mathrm{mg} \mathrm{kg}^{-1}\right)$ & $126 \pm 11,93$ & $37 \pm 0,35$ & $59 \pm 15,10$ & 0,01 & 0,04 \\
\hline $\mathrm{Mn}\left(\mathrm{mg} \mathrm{kg}^{-1}\right)$ & $3.179 \pm 24,29$ & $14.016 \pm 499$ & $903 \pm 1.061$ & 0,37 & 1,22 \\
\hline
\end{tabular}

Os tratamentos envolveram o Argissolo e o Nitossolo sem aplicação de resíduo (dose 0) e combinações dos solos mais o resíduo incorporado, nas doses: 2,5; 5; 10 e 20\%, considerando $10 \mathrm{~cm}$ de profundidade. É importante ressaltar que essa contaminação incorporada retrata uma situação hipotética de contaminação. Caso o resíduo fosse aplicado somente em superfície, os resultados poderiam apresentar falso-negativos, especialmente se os organismos ficassem abaixo da superfície sem ter contato com o resíduo e tempo hábil para oxidá-lo, no caso do teste de fuga.

\section{Parâmetros gerais dos testes}

Para os testes de colêmbolos e minhocas ambos foram montados com cinco réplicas inteiramente casualizados. Todas as réplicas permaneceram em câmara com temperatura controlada a $22^{\circ} \mathrm{C}$, fotoperíodo de $16: 8 \mathrm{~h}$ (luz:escuro) e o $\mathrm{pH}$ e a umidade determinados no início e no final do teste. Previamente o solo foi umedecido a $60 \%$ da capacidade de retenção de água (ISO, 1998a). Para validar os testes foi utilizado o solo artificial tropical (SAT) com 5\% de matéria orgânica (Oliveira Filho et 
al., 2015b). Os espécimes de colêmbolos e minhocas foram obtidos de culturas de laboratório mantidas como descrito nos protocolo ISO 17512-2 (ISO, 2011) e ISO 17512-1 (ISO, 2008), respectivamente.

\section{Avaliação do valor do $\mathrm{pH}$}

Para obter um controle melhor sobre a variação do $\mathrm{pH}$, foi montado um teste para avaliação do valor do $\mathrm{pH}$ em água $(1: 5 \mathrm{~m} / \mathrm{v})$ com cinco réplicas de cada tratamento, onde no início (dia zero) e ao final de dois, 28 e 56 dias foram avaliados, como descrito em Oliveira Filho et al. (2015b).

\section{Teste de fuga com Folsomia candida e Eisenia andrei}

O teste com $F$. candida foi baseado no protocolo da ISO 17512-2 (ISO, 2011), utilizando potes plásticos (diâmetro: $6,5 \mathrm{~cm}$; altura: $5,5 \mathrm{~cm}$ ) divididos em duas seções iguais por uma divisória plástica introduzida verticalmente. Uma seção foi preenchida com $30 \mathrm{~g}$ de solo não contaminado (solo controle) e outra seção com $30 \mathrm{~g}$ de solo contaminado com resíduo. Após a remoção da divisória, 20 colêmbolos (10 a 12 dias de idade) foram introduzidos no centro de cada réplica. Após $48 \mathrm{~h}$ de incubação, o conteúdo de cada seção foi esvaziado para outro pote plástico ao qual foi adicionada água para flutuação dos colêmbolos seguido da contagem dos organismos.

$O$ teste com $E$. andrei foi baseado no protocolo da ISO 17512-1 (ISO, 2008) e segue o mesmo método de montagem descrito para colêmbolos, porém utilizam-se potes maiores (comprimento: $20 \mathrm{~cm}$; largura: $12 \mathrm{~cm}$; altura: $5 \mathrm{~cm}$ ) preenchidos com $250 \mathrm{~g}$ de solo em cada seção e 20 minhocas sincronizadas e cliteladas, previamente lavadas e secas (utilizando papel absorvente). Após incubação por $48 \mathrm{~h}$, o solo foi dividido para contagem das minhocas.

Os protocolos preveem a montagem de um duplo-controle com SAT e com solo controle, no qual tiveram o mesmo procedimento de montagem, porém o mesmo solo não contaminado nas duas seções dos potes plásticos.

\section{Teste de sobrevivência e reprodução com} Folsomia candida e Eisenia andrei

O teste de reprodução com $F$. candida foi baseado no protocolo ISO 11267 (ISO, 1999), com duração de 28 dias. Cada réplica consistiu de um pote plástico (diâmetro: $6,5 \mathrm{~cm}$; altura: $5,5 \mathrm{~cm}$ ), preenchido com $30 \mathrm{~g}$ de cada solo testado, com 10 colêmbolos (10-12 dias de idade). Os colêmbolos foram alimentados no início do teste e aos 7, 14 e 21 dias com fermento biológico. Semanalmente, os potes foram abertos para aeração e controle da umidade (por diferença de peso) pela adição de água. Ao final do teste, o conteúdo do frasco foi esvaziado para outro recipiente e adicionado água, quando necessário foram adicionadas algumas gotas de tinta azul para facilitar a contagem. Após agitar o conteúdo do recipiente, foram tomadas fotos digitais e o número de adultos e juvenis vivos que aparecerem na superfície foram contados usando Software ImageTool 3.0 (University of Texas Health Science Center, San Antonio, EUA).

$O$ teste de reprodução com E. andrei foi realizado de acordo com o protocolo ISO 11268-2 (ISO, 1998b), com duração de 56 dias. Cada réplica consistiu de potes plásticos (diâmetro: 11,5 cm; altura: $9 \mathrm{~cm}$ ), preenchido com $500 \mathrm{~g}$ de cada solo testado, com 10 minhocas sincronizadas e cliteladas, com massa entre 250 a $600 \mathrm{mg}$. As minhocas foram alimentadas no início do teste e a cada semana com 5 $\mathrm{g}$ de esterco úmido de cavalo (desfaunado por congelamento) e com histórico de não utilização de antibiótico durante a vida do animal. Após 28 dias (teste de sobrevivência), as minhocas adultas foram removidas e pesadas, deixando os casulos incubados por mais 28 dias para permitir que os juvenis eclodissem. Ao final de 56 dias (teste de reprodução), os potes plásticos foram colocados em banho-maria a $60{ }^{\circ} \mathrm{C}$ por $40-50$ minutos para forçar a migração dos juvenis a superfície do solo facilitando a contagem.

\section{Análise estatística}

A resposta para o teste de fuga de cada solo foi calculada de acordo com a diretriz da ISO 17512 (ISO, 2008, 2011) utilizando a fórmula: $\mathrm{A}=((\mathrm{C}-$ $\mathrm{T}) / \mathrm{N}) * 100$, onde: $A=$ porcentagem de fuga, $C=$ número de organismos no solo de referência, $T=$ número de organismos no solo contaminado, $N=$ número total de organismos. Sua significância foi avaliada usando o teste de Fisher de one-tail para fuga entre solos controle e contaminados e de two-tail para o teste de duplo-controle (Zar, 1996). Esse procedimento estatístico permite comparar a distribuição de organismos em relação a uma distribuição esperada assumindo a não existência da resposta de fuga para determinado tipo de solo (hipótese nula). A hipótese nula é rejeitada (há 
comportamento de fuga significativo) quando $\mathrm{p}<0,05$. Antes da avaliação de significância, o número de indivíduos foi corrigido usando a taxa de letalidade registrada nos testes.

Valores de $\mathrm{CF}_{50}$ (concentração que origina $50 \%$ de fuga) foram estimados utilizando o software PriProbit ${ }^{\circledR} 1.63$ (Graduate School of Agriculture, Kyoto, Japão). Embora as normas da ISO (ISO, 2008, 2011) utilizem a terminologia CE (concentração estimada) para determinar uma concentração que causa efeitos negativos na reprodução ou fuga de organismos, aqui foi utilizada a terminologia CF como forma de diferenciar da CE com efeitos na reprodução.

Para os testes de fuga, sobrevivência e reprodução e evolução do valor $\mathrm{pH}$, diferenças entre o solo controle e o solo contaminado foram avaliadas através de análise de variância (One-way ANOVA) seguida pelo teste de Dunnett $(\mathrm{M} \leq$ controle, $\mathrm{p}<0,05)$, utilizando o software Statistica 7.0 (Statsoft Inc., Tulsa, EUA).

Valores de $\mathrm{CL}_{50}$ (concentração que causa $50 \%$ de letalidade) foram estimadas utilizando o software PriProbit ${ }^{\circledR} 1.63$ e $\mathrm{CE}_{50}$ (efeitos negativos na reprodução) estimada por análise de regressão não linear, com o software Statistica 7.0.

\section{RESULTADOS}

\section{Características do solo}

Os valores estimados de metais adicionados ao solo considerando a maior dose desse resíduo (20\%) foi de $17,5 \mathrm{mg} \mathrm{kg}^{-1} \mathrm{Cu}, 14,8 \mathrm{mg} \mathrm{kg}^{-1} \mathrm{Zn} \mathrm{e} 226$ $\mathrm{mg} \mathrm{kg}-1 \mathrm{Mn}$, valores estes que ficam abaixo dos teores naturais encontrados no solo (Tabela 1).

$\mathrm{O}$ valor de $\mathrm{pH}$ do Argissolo (dose 0) e do Nitossolo (dose 0) é naturalmente baixo, sendo diferente entre as doses de resíduo aplicadas em cada data avaliada $(\mathrm{p}<0,001)$. Ainda, ocorreu diferença entre datas avaliadas para o mesmo tratamento somente aos 56 dias após aplicação do resíduo piritoso (Dia zero; $\mathrm{p}<0,001$ ) (Tabela 2).

Tabela 2. Valores médios ( \pm desvio padrão, $n=5)$ de pH em água no Argissolo Vermelho Amarelo e Nitossolo Háplico no início (Dia zero) e ao final de dois, 28 e 56 dias.

\begin{tabular}{lllll}
\hline Doses & Dia zero & 2 dias após & 28 dias após & 56 dias após \\
\hline Argissolo & & & & \\
0 & $3,55( \pm 0,03)$ & $3,57( \pm 0,08)$ & $3,90( \pm 0,02)$ & $3,11( \pm 0,09)^{\prime \prime}$ \\
2,5 & $3,32( \pm 0,02)^{*}$ & $3,30( \pm 0,02)^{*}$ & $3,40( \pm 0,01)^{*}$ & $2,47( \pm 0,02)^{* "}$ \\
5 & $3,16( \pm 0,02)^{*}$ & $3,16( \pm 0,01)^{*}$ & $3,11( \pm 0,04)^{*}$ & $1,80( \pm 0,06)^{* *}$ \\
10 & $2,93( \pm 0,01)^{*}$ & $2,93( \pm 0,01)^{*}$ & $2,83( \pm 0,15)^{*}$ & $1,84( \pm 0,09)^{* *}$ \\
20 & $2,54( \pm 0,02)^{*}$ & $2,57( \pm 0,02)^{*}$ & $2,56( \pm 0,09)^{*}$ & $1,73( \pm 0,04)^{* *}$ \\
Nitossolo & & & & \\
0 & $4,48( \pm 0,10)$ & $4,60( \pm 0,09)$ & $4,57( \pm 0,02)$ & $4,20( \pm 0,14)^{\prime \prime}$ \\
2,5 & $4,29( \pm 0,05)^{*}$ & $4,41( \pm 0,01)^{*}$ & $4,15( \pm 0,01)^{*}$ & $3,69( \pm 0,02)^{* \prime}$ \\
5 & $4,00( \pm 0,02)^{*}$ & $4,16( \pm 0,01)^{*}$ & $4,10( \pm 0,01)^{*}$ & $3,59( \pm 0,03)^{* "}$ \\
10 & $3,59( \pm 0,02)^{*}$ & $3,77( \pm 0,02)^{*}$ & $3,81( \pm 0,01)^{*}$ & $3,18( \pm 0,05)^{* "}$ \\
20 & $3,01( \pm 0,02)^{*}$ & $3,22( \pm 0,05)^{*}$ & $3,33( \pm 0,06)^{*}$ & $2,29( \pm 0,03)^{* *}$ \\
\hline
\end{tabular}

Asterisco $(*)$ na coluna difere do respectivo controle (dose 0) e aspas (") na linha difere do respectivo controle (Dia zero) pelo teste de Dunnett $(\mathrm{M} \leq$ controle). Doses do resíduo piritoso: $0=$ controle (sem resíduo); $2,5=2,5 \%$ de resíduo; $5=5 \%$ de resíduo; $10=10 \%$ de resíduo; $20=20 \%$ de resíduo. 


\section{Teste de fuga}

Nos testes de fuga, para F. candida e para E. andrei os duplo-controles não apresentaram diferenças na distribuição ( $p>0,05$ - teste de Fisher) (Figura 1). Todas as concentrações testadas induziram uma resposta de fuga de F. candida e E. andrei em ambos os solos $(\mathrm{p}<0,05)$ (Figura 1). Ainda, observa-se que com o aumento da dose do resíduo piritoso nos dois solos ocorreu um aumento na resposta de fuga de ambas as espécies. Valores estimados de $\mathrm{CF}_{50}$ foram 5,3\% (3,07,5 ) no Argissolo e $<2,5 \%$ (o intervalo de confiança não pode ser calculado) no Nitossolo para $F$. candida. No caso de E. andrei os valores estimados de $\mathrm{CF}_{50}$ foram $<2,5 \%$ (o intervalo de confiança não pode ser calculado) no Argissolo e 8,5\% (6,6-10,2) no Nitossolo.

\section{Sobrevivência e reprodução}

F. candida, apresentou taxa de sobrevivência no solo sem contaminação maior que $90 \%$ e o número médio de juvenis de $110 \pm 32$ e $141 \pm 40$, no Argissolo e no Nitossolo respectivamente (Figura 2a). Já E. andrei, apresentou taxa de sobrevivência de $100 \%$ e o número médio de juvenis de $18 \pm 6$ e $19 \pm$ 9 juvenis, respectivamente no Argissolo e no Nitossolo sem contaminação (Figura 2b).

A sobrevivência de $F$. candida foi afetada no Argissolo $(\mathrm{F}=10,88, \mathrm{p}<0,0001)$ e no Nitossolo $(\mathrm{F}=$ $25,96, \mathrm{p}<0,0001)$, com valores estimados de $\mathrm{CL}_{50}$ de $6,9 \%$ e $3,1 \%$ (os intervalos de confiança não puderam ser calculados), respectivamente (Figura 2a). A sobrevivência de E. andrei, foi afetada no Argissolo (F $=58,63, \mathrm{p}<0,0001)$, entretanto a sobrevivência não foi afetada no Nitossolo ( $F=0,77, \mathrm{p}>0,5)$. Valores estimados de $\mathrm{CL}_{50}$ foram 18,7\% (16,2-22,9) no Argissolo e $>20 \%$ no Nitossolo, neste caso o intervalo de confiança não pode ser calculado (Figura 2b).

A reprodução de $F$. candida e E. andrei foi afetada no Argissolo e Nitossolo em todas as doses avaliadas do resíduo $(\mathrm{p}<0,0001)$ (Figura 2a-b). Ambos os organismos apresentaram baixa reprodução nos solos contaminados, sendo significativamente inferior aos solos controles e, em algumas das doses, não ocorreu reprodução destes organismos. Os valores de $\mathrm{CE}_{50}$ ficaram abaixo de 2,5\% para $F$. candida nos dois solos, enquanto que $E$. andrei apresentou valor de $\mathrm{CE}_{50}$ abaixo de 2,5\% no Argissolo e de 5,5\% (1,3-9,7) no Nitossolo.

\section{DISCUSSÃO}

\section{Efeitos no solo}

Supõe-se que ocorra efeito negativo inicial nos organismos, por exemplo, resposta de fuga (Figura 1) ou baixo número de juvenis nos solos contaminados (Figura 2), por conta do baixo pH, seguido da biodisponibilidade dos metais do resíduo e do solo, visto que ocorre um baixo aporte de metais pela maior dose do resíduo piritoso (20\%) (Tabela 1).

As quantidades de metais estão diretamente relacionadas com os valores de $\mathrm{pH}$ no solo, além disso o valor de $\mathrm{pH}$ influencia na adsorção dos elementos nos sítios do solo, causando maior ou menor toxicidade (van Gestel e Mol, 2003). 

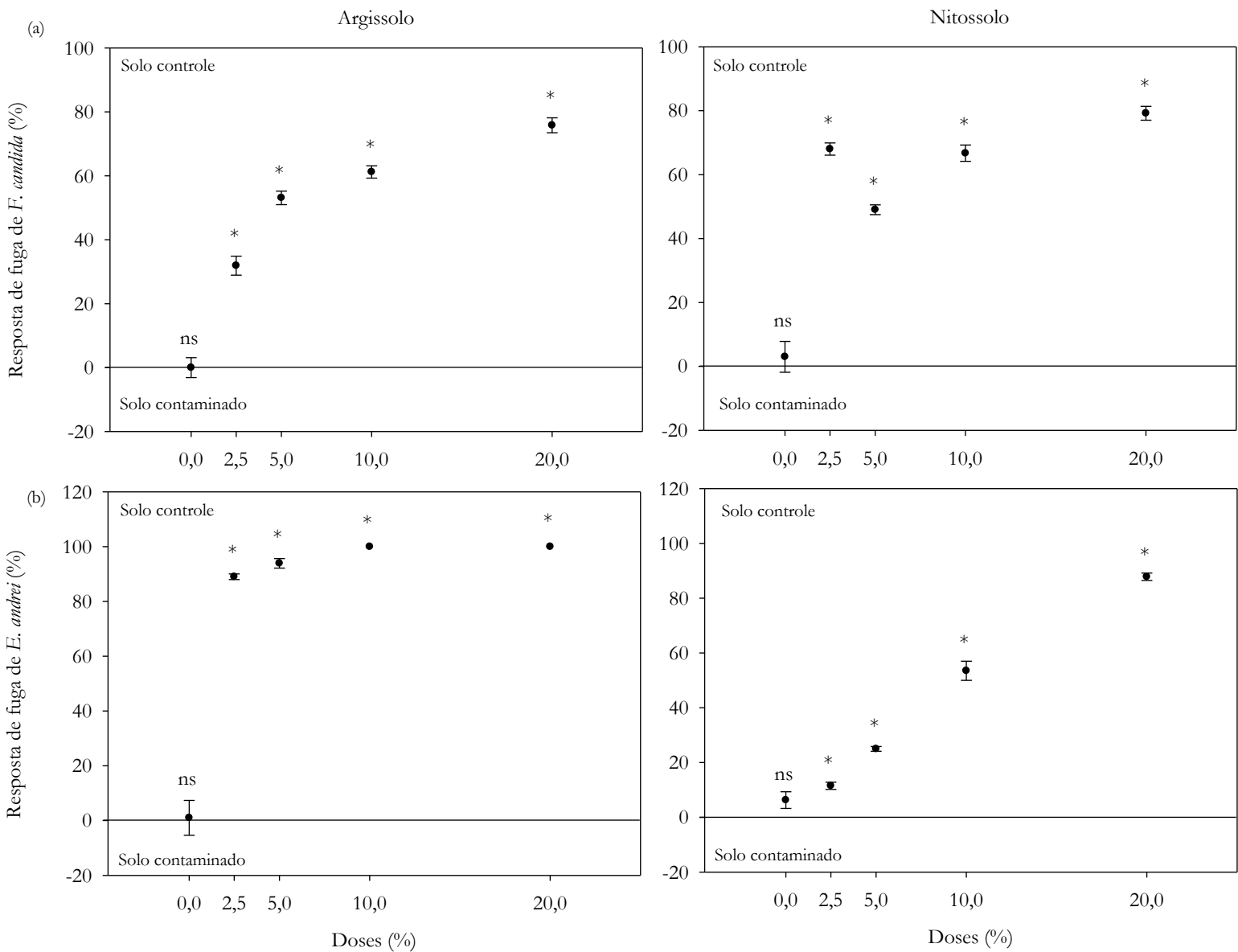

Figura 1. Resposta de fuga (\%) de Folsomia candida (a) e Eisenia andrei (b) quando expostos ao solo controle e solo contaminado nas diferentes doses. $(T)$ desvio padrão $(n=5)$. $*$ significativo, $\mathrm{p}<0,05 ; \mathrm{ns}=$ não significativo, $\mathrm{p}>0,05$, avaliado pelo teste de Fisher. Doses do resíduo piritoso: $0=$ controle (sem resíduo); 2,5 = 2,5\% de resíduo; $5=5 \%$ de resíduo; $10=10 \%$ de resíduo; $20=20 \%$ de resíduo.

A complexidade do ambiente solo faz com que seja difícil de explicar efeitos sobre organismos a partir de efeitos simples de $\mathrm{pH}$ e de concentrações totais de metais. Deve ser ressaltado ainda os efeitos da toxicidade da mistura de metais, bem como da sensibilidade de cada organismo. Oliveira Filho et al. (2015b) apontaram para esse tipo de dificuldade de interpretar e discriminar o efeito da acidificação do solo e biodisponibilidade dos metais. Ainda que nesse estudo os efeitos possam ser atribuídos para efeitos simples de $\mathrm{pH}$, metais ou mistura de metais.

Avaliação de risco ecológico de misturas de metais no solo é dificultada pela complexidade das relações metais-solo-organismo (Calamari e Alabaster, 1980), embora muitos trabalhos usem a contaminação de um único metal. Assim, a extrapolação para misturas, organismos ou condições de exposição é dificultada pela falta de conhecimento sobre a interação das relações metais-solo-organismo.

Os menores valores de $\mathrm{pH}$ resultaram do processo de oxidação da pirita decorrente da exposição do resíduo piritoso no solo por um curto (dois dias após a aplicação) e longo tempo (56 dias após a aplicação) (Tabela 2). Daniels (1996) relata que em questão de alguns meses o $\mathrm{pH}$ pode passar de 7,5 para valores menores do que $3,0 \mathrm{em}$ solos contaminados com pirita. Aqui, a maior diferença entre o valor inicial (Dia zero) e final (após 56 dias) de $\mathrm{pH}$ foi no Argissolo na dose 5, em que a diferença foi de 1,36 unidades do valor de $\mathrm{pH}$. Embora o valor da diferença seja baixo, ele foi suficiente para prejudicar a reprodução dos organismos (Figura 2). 

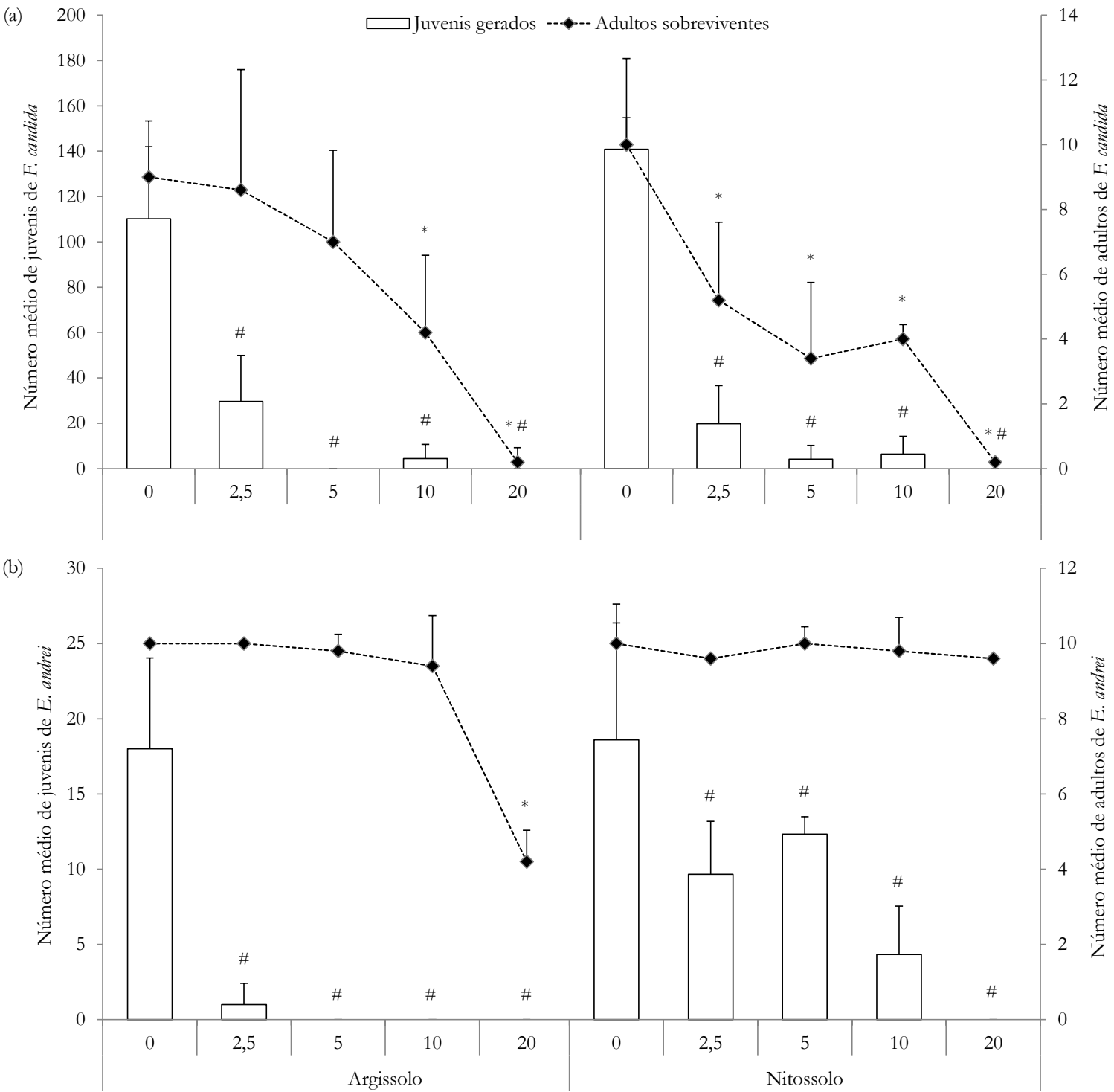

Figura 2. Sobrevivência e reprodução de Folsomia candida (a) e Eisenia andrei (b) quando expostos ao solo controle e contaminado. $(T)$ desvio padrão $(n=5)$ * e \#: significativo para número médio de adultos e juvenis, respectivamente, p $<0,05$, avaliado pelo teste de Dunnett. Doses do resíduo piritoso: $0=$ controle (sem resíduo); $2,5=2,5 \%$ de resíduo; 5 $=5 \%$ de resíduo; $10=10 \%$ de resíduo; $20=20 \%$ de resíduo.

\section{Efeitos nos organismos}

Como um primeiro teste avaliando a função de hábitat do solo, o comportamento de fuga (toxicidade aguda) apontou toxicidade dos dois solos (Figura 1), em que os organismos edáficos evitaram os solos contaminados com o resíduo piritoso. A baixa letalidade $(<9 \%)$ indica que os níveis de resíduos incorporados ao solo tiveram pouquíssima influência sobre a letalidade de colêmbolos e minhocas em curto prazo. Os resultados obtidos no teste de fuga com F. candida diferiam dos obtidos com E. andrei, provavelmente porque os colêmbolos estão menos expostos a solução do solo do que as minhocas. Ao contrário de outros organismos 
(plantas, enquitreídeos, minhocas), que estão em contato direto com a solução do solo, os colêmbolos, especialmente da espécie $F$. candida vivem principalmente na superfície do solo ou em poros de grandes dimensões que são normalmente drenados (FROUZ et al., 2011). Além disso, são cobertos por uma cutícula com permeabilidade limitada aos líquidos, assim, $\mathrm{pH}$ e substâncias químicas na solução do solo são capazes de apresentar um menor efeito direto sobre os colêmbolos do que em outros organismos (FROUZ et al., 2011). Comportamento de fuga de áreas contaminadas é considerado como um sinal de alerta precoce para os solos poluídos (Natal da Luz et al., 2004) e provêm informações sobre a capacidade dos organismos em detectar os contaminantes, refletindo em um mecanismo de defesa evitando a exposição crônica (Hellou, 2011) e a morte. Por ser uma estratégia eficiente de sobrevivência dos organismos, a resposta de fuga em larga escala pode ter um impacto negativo sobre a densidade populacional e estrutura da comunidade de organismos edáficos, afetando os ciclos de nutrientes e decomposição de material orgânico (Edwards e Coulson, 1992), induzindo a uma diminuição da qualidade do solo e dificultando o seu processo de recuperação.

Os testes de toxicidade crônica (reprodução) com colêmbolos e minhocas, demonstraram níveis elevados de toxicidade pelo resíduo piritoso com $\mathrm{CE}_{50}$ abaixo de 5,5\%, sendo que ambos os solos apresentaram sensibilidades semelhantes para colêmbolos (Figura 2). As minhocas no Nitossolo apresentaram valores mais elevados na taxa de reprodução que no Argissolo, embora que ainda a função de hábitat do solo esteja comprometida, visto que os solos contaminados foram diferentes do controle. Testes crônicos, de maneira geral, são observados efeitos subletais, que permitem a sobrevivência, mas afetam uma ou mais funções biológicas, como reprodução (Magalhães e FerrãoFilho, 2008). Ainda, esse fato pode estar relacionado com os teores totais originais de $\mathrm{Cu}$ e $\mathrm{Zn}$ serem mais baixos no Nitossolo em relação ao Argissolo (Tabela 1) ou com o valor de $\mathrm{pH}$ um pouco mais elevado no Nitossolo no final do teste (Tabela 2).

Dados de toxicidade disponíveis na literatura para $\mathrm{Cu}, \mathrm{Zn}$ e $\mathrm{Mn}$, utilizando comportamento de fuga, sobrevivência e reprodução, apresentam uma variação considerável, devido principalmente a diferenças nas formas de substâncias utilizadas, parâmetro tóxico avaliado, tipos de solos (alguns estudos fazem uso de solos naturais contaminados em laboratório, solos naturais com contaminação in situ por ação antropogênica e solos artificiais), variações de $\mathrm{pH}$, teor de matéria orgânica, temperatura e umidade.

Segundo Boiteau et al. (2011), $\mathrm{EC}_{50}$ de 18 $\mathrm{mg} \mathrm{Cu} \mathrm{kg}{ }^{-1}$ de solo foram suficientes para promover resposta de fuga em F. candida e de $181 \mathrm{mg} \mathrm{Cu} \mathrm{kg}^{-1}$ de solo para E. andrei (Loureiro et al., 2005). Entretanto, esses valores aumentam para teste de reprodução, em que Greenslade e Vaughan (2003) encontraram EC 50 de $751 \mathrm{mg} \mathrm{Cu} \mathrm{kg} \mathrm{kg}^{-1}$ de solo para F. candida. Nesse estudo, os valores de cobre ficaram entre $26 \mathrm{mg} \mathrm{kg}^{-1}$ para o Nitossolo e $108 \mathrm{mg} \mathrm{kg}^{-1}$ para o Argissolo, o que parece terem tido influência somente na resposta de fuga dos organismos.

Para zinco, Jensen e Pedersen (2006), estudando um solo natural contaminado artificialmente, encontraram valores de $\mathrm{EC}_{50}$ de 184 $\mathrm{mg} \mathrm{Zn} \mathrm{kg-1}$ que foram suficientes para ocasionar uma diminuição na reprodução de F. candida. Nenhum dos valores de $\mathrm{Zn}$ encontrados nos solos estudados e no resíduo piritoso foram suficientes para ocasionar diminuição na reprodução desses organismos.

No caso do manganês, os valores encontrados nos solos (Tabela 1) foram suficientes para influenciar a sobrevivência de F. candida e E. andrei, onde, de acordo com Kuperman et al. (2004), valores de $\mathrm{EC}_{50} 2.575$ e $927 \mathrm{mg} \mathrm{Mn} \mathrm{kg}^{-1}$ de solo, respectivamente, diminuíram a sobrevivência desses organismos. E, segundo o mesmo autor, valores de 1.663 e $927 \mathrm{mg} \mathrm{Mn} \mathrm{kg} \mathrm{kg}^{-1}$ de solo, interferem na reprodução de F. candida e E. andrei, respectivamente.

A sensibilidade dos organismos usados nos testes é de grande importância. Já que a toxicidade pode ser menor para certos organismos (por exemplo minhocas), devido a diferenças de fisiologia animal (e sensibilidade) (Natal-da-Luz et al., 2011). Isso confirma a necessidade de incluir organismos de diferentes níveis tróficos nos testes (Oliveira Filho et al., 2015b), bem como incluir organismos nativos aumentando assim a relevância ecológica.

\section{CONCLUSÕES}

O resíduo piritoso (carvão mais a pirita) afeta negativamente os organismos do solo (Folsomia candida e Eisenia andrei), tanto em curto período de tempo (teste de fuga) quanto em longo prazo (sobrevivência e reprodução).

A capacidade dos colêmbolos e das minhocas em evitar solos contaminados (teste de fuga) com resíduo piritoso é sensível em demonstrar o potencial de danos quanto reprodução. 
No caso de ocorrer um descarte inapropriado desse resíduo ele pode causar a perda do hábitat e da abundância e biodiversidade de colêmbolos e minhocas. Esse estudo também fornece informações ecotoxicológicas sobre a adição de resíduo piritoso seguido da diminuição do $\mathrm{pH}$ e da biodisponibilidade dos metais que podem ser úteis em avaliações de risco ecológico de áreas ligadas a mineração de carvão.

\section{AGRADECIMENTOS}

À Coordenação de Aperfeiçoamento de Pessoal de Nível Superior (CAPES), pela concessão de bolsa de doutorado do primeiro autor. D. Baretta agradece ao Conselho Nacional de Desenvolvimento Científico e Tecnológico (CNPq) pela Bolsa de Produtividade Científica (Processo no 307162/2015$0)$.

\section{REFERÊNCIAS}

ALVARENGA, P.; PALMA, P.; VARENNES, A.; CUNHA-QUEDA, A. C. A contribution towards the risk assessment of soils from the São Domingos Mine (Portugal): Chemical, microbial and ecotoxicological indicators. Environmental Pollution, v. 161, p. 50-56, 2012.

ARNOLD, R. E.; HODSON, M. E. Effect of time and mode of depuration on tissue copper concentrations of the earthworms Eisenia andrei, Lumbricus rubellus and Lumbricus terrestris. Environmental Pollution, v. 148, p. 21-30, 2007.

BHUIYAN, M. A. H.; PARVEZ, L.; ISLAM, M. A.; DAMPARE, S. B.; SUZUKI, S. Heavy metal pollution of coal mine-affected agricultural soils in the northern part of Bangladesh. Journal of Hazardous Materials, v. 173, p. 384-392, 2010.

BOITEAU, G.; LYNCH, D. H.; MACKINLEY, P. Avoidance tests with Folsomia candida for the assessment of copper contamination in agricultural soils. Environmental Pollution, v. 159, p. 903-906, 2011.

CALAMARI, D.; ALABASTER, J. S. An approach to theoretical models in evaluating the effects of mixtures of toxicants in the aquatic environment. Chemosphere, v. 9, p. 533-538, 1980.
CHAPMAN, P. M. Integrating toxicology and ecology: putting the "eco" into ecotoxicology. Marine Pollution Bulletin, v. 44, p. 7-15, 2002.

DANIELS, W. L. Manipulating the chemical properties of soil and mining wastes. In: ALVAREZ, V. V. H.; FONTES, L. E. F.; FONTES, M.P.F. (Eds.). O solo nos grandes domínios morfoclimáticos do Brasil e o desenvolvimento sustentado. Viçosa: Sociedade Brasileira de Ciência do Solo e Universidade Federal de Viçosa, 1996. p. 869-897.

EDWARDS, P.J.; COULSON, J.M. Choice of earthworm species for laboratory tests. In: GREIGSMITH, P. W.; BECKER, H.; EDWARDS, P. J.; HEIMBACH, F. (Eds.). Ecotoxicology of Earthworms. Andover: Intercept, 1992. p. 36-43.

FRANCISCONI, M. S.; BONA, A.; SILVEIRA, F.Z.; GEREMIAS, R.; PICH, C.T. Use of coal mining waste for river water treatment and evaluation of this process using physicochemical parameters and bioassays. Ecotoxicology and Environmental Contamination, v. 8, p. 45-51, 2013.

FROUZ, J.; HRČKOVÁ, K.; LANA, J.; KRIŠTƯFEK, S.; MUDRÁK, O.; LUKEŠOVÁ, A.; MIHALJEVIČ, M. Can laboratory toxicity tests explain the pattern of field communities of algae, plants, and invertebrates along a toxicity gradient of post-mining sites? Applied Soil Ecology, v. 51, p. 114121, 2011.

GEE, G. W.; BAUDER, J.W. Particle size analysis. In: KLUTE, A. (Ed.). Methods of soil analysis Part 1 Physical and mineralogical methods. 2nd ed. Madison: American Society of Agronomy and Soil Science Society of America, 1986. p.383-411.

GEREMIAS, R.; BORTOLOTTO, T.; WILHELMFILHO, D.; PEDROSA, R. C.; FÁVERE, V. T. Efficacy assessment of acid mine drainage treatment with coal mining waste using Allium cepa $\mathrm{L}$. as a bioindicator. Ecotoxicology and Environmental Safety, v. 79, p. 116-121, 2012.

GREENSLADE， P.; VAUGHAN， G. T. A comparison of collembola species for toxicity testing of Australian soils. Pedobiologia, v. 47, p. 171-179, 2003.

HELLOU, J. Behavioural ecotoxicology, an "early warning" signal to assess environmental quality. 
Environmental Science and Pollution Research, v. 18, p. 1-11, 2011.

HOPKIN, S. P. Biology of the Springtails (Insecta: Collembola). Oxford: Oxford University Press, 1997.

ISO International Organization for Standardization. ISO 11274. Soil quality - Determination of the waterretention characteristic - Laboratory methods. Geneva: ISO, 1998a.

ISO International Organization for Standardization. ISO 17512-2. Soil quality - Avoidance test for testing the quality of soils and effects of chemicals - Part 2: Test with collembolans (Folsomia candida). Geneva: ISO, 2011.

ISO International Organization for Standardization. ISO 17512-1. Soil quality - Avoidance test for testing the quality of soils and toxicity of chemicals - Part 1: Test with earthworms (Eisenia foetida and Eisenia andrei). Geneva: ISO, 2008.

ISO International Organization for Standardization. ISO 11267. Soil quality - Inhibition of reproduction of Collembola (Folsomia candida) by soil pollutants. Geneva: ISO, 1999.

ISO International Organization for Standardization. ISO 11268-2. Soil quality - Effects of pollutants on earthworms (Eienia fetida) - Part 2: Determination of effects on reproduction. Geneva: ISO, 1998b.

JENSEN, J.; PEDERSEN, M. B. Ecological risk assessment of contaminated soil. Reviews of Environmental Contamination and Toxicology, v. 186, p. 73-105, 2006.

KOSTARELOS, K.; GAVRIEL, I.; STYLIANOU, M.; ZISSIMOS, A. M.; MORISSEAU, E.; DERMATAS, D. Legacy Soil Contamination at Abandoned Mine Sites: Making a Case for Guidance on Soil Protection. Bulletin of Environmental Contamination and Toxicology, v. 94, p. 269-274, 2015.

KUPERMAN, R. G.; CHECKAI, R. T.; SIMINI, M.; PHILLIPS, C. T. Manganese toxicity in soil for Eisenia fetida, Enchytraeus crypticus (Oligochaeta), and Folsomia candida (Collembola). Ecotoxicology and Environmental Safety, v. 57, p. 48-53, 2004.

LOUREIRO, S.; SOARES, A. M. V. M.;
REVISTA SCIENTIA AGRARIA

Versão On-line ISSN 1983-2443

Versão Impressa ISSN 1519-1125

SA vol. $18 \mathrm{n}^{\circ} .1$ Curitiba Jan/Mar. 2017 p. 64-75
NOGUEIRA, A. J. A. Terrestrial avoidance behaviour tests as screening tool to assess soil contamination. Environmental Pollution, v. 138, p. 121-131, 2005.

LORTZIE, K.; STYLIANOU, M.; DERMATAS, D.; KOSTARELOS, K. Long-term environmental impact at an abandoned gold-silver enrichment plant: A case study in Mitsero, Cyprus. Engineering Geology, v. 184, p. 119-125, 2015.

LUNARDI NETO, A.; ALBUQUERQUE, J. A.; ALMEIDA, J.A.; MAFRA, Á. L.; MEDEIROS, J. C.; ALBERTON, A. Atributos físicos do solo em área de mineração de carvão influenciados pela correção da acidez, adubação orgânica e revegetação. Revista Brasileira de Ciência do Solo, v. 32, p. 1379-1388, 2008.

MAGALHÃES, D. D. P.; FERRÃO-FILHO, A. S. A ecotoxicologia como ferramenta no biomonitoramento de ecossistemas aquáticos. Oecologia Brasiliensis, v. 12, p. 355-381, 2008.

NATAL DA LUZ, T.; RIBEIRO, R.; SOUSA, J. P. Avoidance tests with Collembola and earthworms as early screening tools for site-specific assessment of polluted soils. Environmental Toxicology and Chemistry, v. 23, p. 2188-2193, 2004.

NATAL-DA-LUZ, T.; OJEDA, G.; PRATAS, J.; VAN GESTEL, C. A. M.; SOUSA, J. P. Toxicity to Eisenia andrei and Folsomia candida of a metal mixture applied to soil directly or via an organic matrix. Ecotoxicology and Environmental Safety, v. 74, p. 1715-1720, 2011.

OLIVEIRA FILHO, L. C. I.; BARETTA, D.; VIAPIANA, C. M.; SANTOS, J. C. P. Mesofauna de solo construído em área de mineração de carvão. Revista de Ciências Agroveterinárias, v. 14, p. 55-64, 2015a.

OLIVEIRA FILHO, L. C. I.; BARETTA, D.; ZORTÉA, T.; CASAROTTO, K.; DORS, P.; CAMPOS, M. L.; SANTOS, J. C. P. Avaliação ecotoxicológica do resíduo de mineração de carvão. Revista Brasileira de Ciência do Solo, v. 39, p. 18061813, 2015b.

OLIVEIRA, M. L. S.; WARD, C. R.; FRENCH, D.; HOWER, J. C.; QUEROL, X.; SILVA, L. F. O. Mineralogy and leaching characteristics of 
beneficiated coal products from Santa Catarina, Brazil. International Journal of Coal Geology, v. 94, p. 314-325, 2012.

POMPÊO, M. L. M.; MOSCHINI-CARLOS, V.; ALEXANDRE, N. Z.; SANTO, E. Qualidade da água em região alterada pela mineraç ão de carvão na microbacia do rio Fiorita (Siderópolis, Estado de Santa Catarina, Brasil). Acta Scientiarum - Biological Sciences, v. 26, p. 125-136, 2004.

RODRIGUEZ-IRURETAGOIENA, A.; VALLEJUELO, S. F.-O.; GREDILLA, A.; RAMOS, C. G.; OLIVEIRA, M. L. S.; ARANA, G.; DIEGO, A.; MADARIAGA, J. M.; SILVA, L. F. O. Fate of hazardous elements in agricultural soils surrounding a coal power plant complex from Santa Catarina (Brazil). Science of the Total Environment, v. 508, p. 374-382, 2015.

SILVA, L. F. O.; MACIAS, F.; OLIVEIRA, M. L. S.; BOIT, M. K.; WAANDERS, F. Coal cleaning residues and $\mathrm{Fe}$-minerals implications. Environmental Monitoring and Assessment, v. 172, p. 367-378, 2011.

SIMMONS, J. A. Phosphorus removal by sediment in streams contaminated with acid mine drainage. Water, Air, and Soil Pollution, v. 209, p. 123-132, 2010.

SOARES, E. R. Mobilização de metais pesados em materiais provenientes do complexo carboenergético de Candiota-RS. 1995. 95 p. Dissertação (Mestrado em Agronomia) - Universidade Federal de Viçosa, Viçosa, 1995.

TEDESCO, M. J.; GIANELLO, C.; BISSANI, C. A.; BOHNEN, H.; VOLKWEISS, S. J. Análises de solo, plantas e outros materiais. $2^{\mathrm{a}}$ ed. Porto Alegre: Universidade Federal do Rio Grande do Sul, 1995. (Boletim técnico, 5).

USEPA United States Environmental Protection Agency. Method 3051a: Microwave assisted acid digestion of sediments, sludges, soils, and oils. [internet]. EPA Publication; 2007. p. 1-30. Disponível em:

http://www.epa.gov/osw/hazard/testmethods/sw84 6/pdfs/3051a.pdf.

VAN GESTEL, C. A. M.; MOL, S. The influence of soil characteristics on cadmium toxicity for Folsomia candida (Collembola: Isotomidae). Pedobiologia, v. 47, p. 387-395, 2003.

ZAR, J. Biostatistical Analysis. 3rd ed. London: Prentice-Hall, 1996. 